\title{
Scientific Basis for Deplorable Women's Behaviour: A Connection with Hormonal Imbalance
}

\author{
Jeetendra Kumar Gupta*, Ahsas Goyal, Bhavini Saraf and Kajal Thareja \\ Institute of Pharmaceutical Research, GLA University Mathura, Chaumuhan - 281406, Uttar Pradesh, India; \\ jkgupta81@rediffmail.com, ahsasgoyal1990@gmail.com, bhavini.saraf@gmail.com, kajalthareja8@gmail.com
}

\begin{abstract}
Objectives: Etiquette and behaviour depend on hormonal ambiance. This communication renders a link between women's behaviour which deviates from normal because of certain hormonal imbalance. Methods/Analysis: Interpretation of women's behaviour was accomplished on the basis of existing literatures and researches. Various endogenous molecules such as serotonin, dopamine, oxytocin, endorphins, prolactin and cortisol have diversified effects on mood and behaviour. Findings: The emergence of anxiety, anger, stress, depression or any psychotic change has irrefutable relationship with certain endogenous molecule. With the help of existing literatures and researches, it has been inferred that deficiency of serotonin is a leading cause of depression, while dopamine deficiency can plunk a woman into non-impulsive, emotionless behaviour. Excess of cortisol can lead to the feeling of stress while hyperprolactinemia causes prodigious production of milk even in non-pregnant and non-nursing women. It also causes disturbance in libido and irregular menstrual cycles. Endorphins are endogenous neuropeptides that reduce pain and boost pleasure. Improvement: The article apprehends a connection between conduct and hormonal framework. A healthy lifestyle can ameliorate the hormonal ambiance; thereby a break through improvement in women's behaviour can be interposed.
\end{abstract}

Keywords: Depression, Hormonal Imbalance, Libido, Stress, Women's Behaviour

\section{Introduction}

The contingency of mood disorder in women is about two times higher than men. The underlying reality of this gender variance is not yet understood completely 1 . The word 'emotion' is derived from the French word 'emouvoir' which means 'to stir up. Emotion is a mental state which plays a prime role in human life and immensely associated with physiological and neurobiological activities. The limbic system is a complex set of structures that plays a significant role in processing emotions in an individual. Amygdala is a salient structure of limbic system that assists in detecting and preparing emergency events associated with emotion 2 . Amygdala and prefrontal cortex are the parts of brain which regulate emotions of well being. Amygdala is an almond shaped structure helps in the function via clusters of nuclei present at the anterior medial portion of each temporal lobe ${ }^{3}$. The prefrontal cortex located at the anterior portions of cerebral cortex which regulates the release of neurotransmitters, such

*Author for correspondence as dopamine, serotonin and norepinephrine which are essential for mood regulation ${ }^{4}$.

Emotional changes start occurring from early maturity to old age. Earlier it was postulated that with aging people became depressed and feels loneliness. In contrast it has been now observed by many researchers that with aging the rational outlook towards things increases, which means that aged people have more sensible outlook than younger people. The emotion is considerately coherent to arousal of the nervous system. Arousal of nervous system with stimuli regulate with aging. A high degree of differences in positive arousal response in case of younger people as compared to aged people has been observed. Aging led to decline in arousal response due to atrophy of amygdala ${ }^{5}$.

Women are more emotional as compared to men in respect of their emotional expressivity and emotional experience. Emotional experience refers to individual arousal by an extrinsic stimuli and emotional expressivity refers to extrinsic expression of individual experience. 
There are many types of emotions, such as sadness, anger, horror, disgust, neutrality, surprise, amusement and pleasure. Sex difference in emotional experience and emotion expressivity depend on distinct types of emotions ${ }^{6}$.

Hormones are the chemical substances released from glands leading to change in behaviour of individual. Serotonin, dopamine, endorphins, oxytocin, prolactin and cortisol regulate individual's mood and behaviour, while gonadal hormones like estradiol and testosterone have also influential role ${ }^{7}$. Any imbalances among those hormones can lead to profound change in behaviour.

\section{Hormones and Mood}

The limbic system of brain regulates emotions in an individual. It is immensely influenced by the activity of autonomic and endocrine system. Amygdala processes most of the emotions. The limbic system shows interaction via congulate gyrus to prefrontal cortex ${ }^{8}$.

Hormones are the primary messengers that influence behaviour of an individual by regulating, integrating and controlling physiological functions. Sex hormones also control the behavioural changes in an individual. The other primary messengers which regulate the humour of an individual are serotonin, dopamine and oxytocin, although prolactin, cortisol and endorphins have also indirect role. Happiness is the sentiment of complete satisfaction and pleasure in a person. The hormones which are liable for happiness are Serotonin, dopamine and oxytocin $\%$. These are the chemical messengers realised from specific glands into the circulatory system to target the particular site in order to induce contentment and pleasure in an individual.

Serotonin: It is a monoamine neurotransmitter derived from essential amino acid tryptophan by hydroxylation and subsequent decarboxylation. It is produced from raphe nuclei in brain stem, enterochromaffin cells of gastro intestinal tract as well as from the platelet cells. It is metabolized by liver into active metabolite 5-Hydroxyindoleacetic acid (5-HIAA). The 5-HT receptors are categorised into many types having 7 major classes $\left(5-\mathrm{HT}_{1} \text { to } 5-\mathrm{HT}_{7}\right)^{10}$. Except $5-\mathrm{HT}_{3}$ receptor, all are G-protein coupled receptor where as $5-\mathrm{HT}_{3}$ is a ligand gated ion-channel receptor. Serotonin elevate mood and used as an efficient target in the therapy of depression and anxiety. The selective serotonin reuptake inhibitors are approved by Food Drug and Administration and considered as an effective approach for the treatment of depression. The drugs like fluoxetine, Citalopram and Sertraline are some commonly used medications as antidepressant ${ }^{11}$. In an experiment, it has been seen that some extent of shining light elevates the level of serotonin in an individual employed in the treatment of seasonal depression. Physical activities also elevate the level of 5-HT in an individual. Active lifestyle shackles the biosynthesis of branched chain amino acids which are isoleucine, luecine and valine, elevating the level of 5-HT and the diet loaded with essential amino acid tryptophan also elevate the level of 5-HT르.

Dopamine: It is a contraction of 3,4dihydroxyphenylethylamine which plays an essential role in the physiology of brain and body ${ }^{13}$. It is a monoamine neurotransmitter synthesized in substantial nigra, ventral and tegmental region. Dopamine is synthesized from essential amino acid phenylalanine and nonessential amino acid tyrosine. It is metabolized by cathechol-omethyl transferase, monoamine oxidases and aldehyde dehydrogenase to homovanillic acid as final product which is excreted in urine. Dopamine receptors are G-protein coupled receptors classified into five types $\left(D_{1} \text { to } D_{5}\right)^{14}$. Mesocorticolimbic, nigrostriatal, tuberoinfundibular and hypothalamospinal are the dopaminergic projections of neurons located in central nervous system to release the neurotransmitter dopamine. The mesolimbic projection of Mesocorticolimbic pathway releases dopamine from ventral tegmental area present in midbrain responsible for reward and pleasure response. Reward system of brain is an organized group of neural structures mainly responsible for incentive salience, associative learning and positive emotions such as joy, ecstasy and euphoria. The reward system is greatly linked with elevated level of dopamine in an individual in order to feel pleasure ${ }^{15}$. The depression also occurs when reward pathway prediction errors ${ }^{16}$.

Oxytocin: It is a peptide hormone synthesized in supraoptic and para ventricular nuclei of hypothalamus ${ }^{17}$. This hormone is responsible for social attachment, parturition, motherly and etiquette, anti-stress and antiaggression activities. It is referred to as love hormone also ${ }^{\frac{18}{8}}$. It has influential role on excitatory-inhibitory equilibrium. The anti-anxiety effect of oxytocin was estimated by elevated plus maze method on rodents. The elevated level of oxytocin leads milk ejection from mammary gland in lactating women. 
Endorphins: Endorphins are predominately analogous to the structure of morphine. It is a word which has been generated by 'endogenous' i.e., from the body and morphine which is a pain reliever. Endorphins are the neurochemicals that are released by the brain's hypothalamus and the pituitary gland in a condition when the human body experiences pain such as labour pain or is under any kind of physical stress ${ }^{19}$. Endorphins act on the opioid receptors in the brain. They are regarded as natural opioids. Endorphins have metabolic as well as hormonal respond to exercises or trainings. In cases of exercise the level of $\beta$-endorphin and $\beta$-lipotrophin gets elevated and their effects are directly related to psychological and physiological modifications which may include changes in mood, euphoria and stress. Endorphins also play an important role for our feeling of pleasure and happiness ${ }^{20}$. Affection or memories are controlled by the brain's limbic system, which may generally include the hypothalamus that operates the functioning of body such as breathing and satisfaction to hunger and emotional response. Obsessive Compulsive Disorder (OCD) is a mental disorder which can occur due to decreased level of endorphins ${ }^{21}$.

Prolactin: Luteotropic hormone or luteotropin are the other names of prolactin hormone. Pituitary gland is responsible for the secretion of prolactin. Prolactin binds in central nervous system in the hypothalamus, choroid plexus and in substantia nigra region 22 . Prolactin is generally linked with milk production in mammals. Thyrotrophic releasing hormone stimulates the release of prolactin. It enhances the lactation due to the increment in serum concentrations amidst the pregnancy that leads to the aggrandizement of the mammary glands. Dopamine is a neurotransmitter which is responsible for the inhibition of prolactin ${ }^{23}$. The elevated levels of prolactin in human body leads to the decline in the levels of estrogen in females and testosterone in males ${ }^{24}$. Over production of prolactin due to a non-cancerous tumour leads to a condition known as hyperprolactinemia ${ }^{25}$. In females, hyperprolactinemia leads to oligomennorhea or amenorrhea, galactorrhea (when not pregnant), vaginal dryness that may cause painful copulation and hirsutism while in males elevated levels of prolactin may lead to erectile dysfunction and gynecomastia ${ }^{26}$. Hyperprolactinemia decreases the level of sexual behaviour in mammals i.e., decreased libido. Some psychological disturbances are also related to hyperprolactinemia which include anxiety, depression and somatization etc. Bromocriptine and pergolide are used to maintain the level of prolactin in case of adverse situations $^{27}$. Central nervous system has a great impact of prolactin which may lead to behavioural changes. Some other complications related to prolactinoma are vision loss, hypopituitarism, osteoporosis and pregnancy complication $s^{28}$.

Cortisol: Chronic stress is affiliated with psychological disturbances which generally includes depression and anxiety. Cortisol hormone is responsible for stress ${ }^{29}$. It is also known as $(11 \beta, 17 \alpha)$-21-trihydroxypregn-4-ene3,20 -dione ${ }^{30}$. Cortisol is a glucocorticoid hormone which is originated by the zona-fasciculata of adrenal cortex in the adrenal gland. It is also responsible for suppressing the immune system of the body by increasing the blood sugar levels $\mathrm{s}^{\underline{31}}$. Elevated level of cortisol may lead to depression, obesity, low level of libido, acne, insomnia, blood sugar dysregulation, hypertension, elevated level of cholesterol and heart diseases. Cortisol respond to stress or fear is also linked with negative mood stress. Excessive cortisol secretion by the adrenal gland produces Cushing's syndrome. Glucocorticoids generally have impact on the emotion related regions of the brain. It affects the memory and learning processes and also stimulates the gastric acid secretion. In some studies it has been seen that the elevated level of cortisol may be lowered by administrating phosphatidylserine, magnolia bark extract and ashwagandha extract. Cheerful lifestyle also helps to lower the cortisol level ${ }^{32}$.

Apart from these endogenous chemicals, sex hormones such as estrogens and testosterone have also

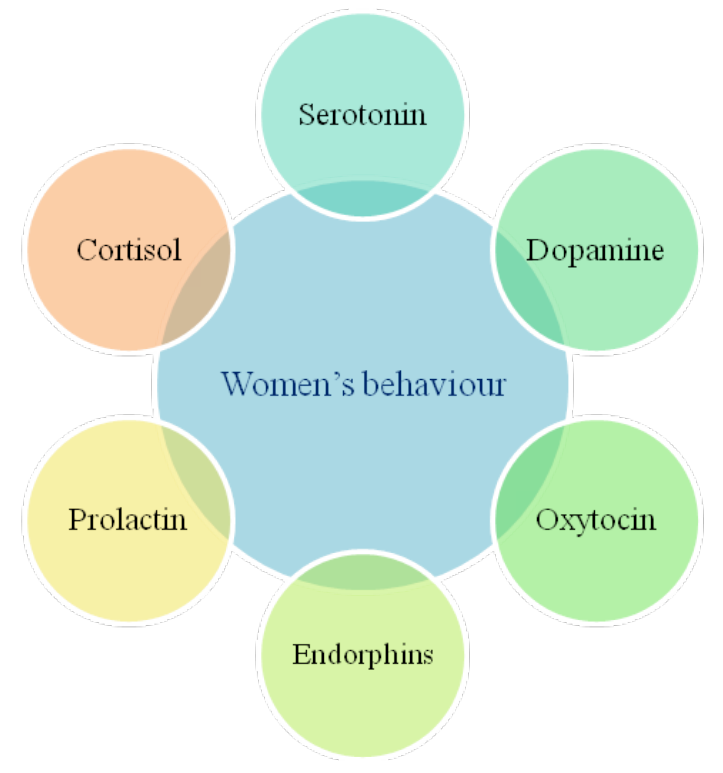

Figure 1. Pivotal chemicals that influence women's behaviour apart from sex hormones. 
influential role in emotional behaviour and women's aggression. It has been seen that estrogens improve mood whereas excess of testosterone in women is a leading cause of aggressive behaviour (Figure 1).

\section{Hormonal Imbalance and its Effect on Behaviour}

Deficiency of endorphin may lead to depression and chronic pain. Endorphin Deficiency Disorder (EDS) may have symptoms such as manic depression and bipolar disorder. Its deficiency may also cause aches and pain especially in the neck and back, trouble sleeping and impulsive behaviour ${ }^{33}$.

Excessive generation of prolactin in the blood circulation may cause hyperprolactinemia. Symptoms due to excessive production or release include unusual milk production, amenorrhoea and decrease in levels of sex hormones, such as estrogen in females and testosterone in males ${ }^{34}$. Deficiency of prolactin may lead to hypoprolactinemia. It generally occurs in those people who have pituitary under activity. Hypoprolactinemia may cause insufficient milk production after giving birth.

Unbalanced cortisol in the body may cause anxiety, irritability, insomnia, decreased libido, loss of muscle mass and lower bone density ${ }^{35}$. If the body tissues are exposed to cortisol for a longer time span it may lead to Cushing's syndrome and if the adrenal gland is unable to manufacture significant amount of cortisol it may cause Addison's disease ${ }^{36}$. Elevated cortisol levels may also lead to stimulate the appetite and cause obesity and also hypertension while opposite effect could be seen when level of cortisol declines. Imbalance in cortisol leads to complications in pregnancy ${ }^{37}$.

Dopamine imbalance could influence the mood, sleep, learning, focus and memory in a human body. Deficiency of this neurotransmitter may lead to depression and Parkinson's disease. If the level of dopamine declines it may generate symptoms such as muscle cramps, stiffness in muscles, pain, Gastroesophageal reflux disease, low sexual drive, hallucination, delusion etc. It may also cause mental illness such as schizophrenia and psychosis ${ }^{38}$. Pleasure seeking behaviour also known as hedonism could also be seen in people who have excessive level of dopamine. Other symptoms linked to excessive secretion and release of dopamine include social seeking, reward seeking behaviour, person may remain highly motivated, mania or hypomania, hyperactivity, high sexual desire and anxiety ${ }^{39}$.
In females, generally the level of oxytocin declines after menopause. Symptoms associated with the deficiency of oxytocin may include early menopause, hypothyroidism, depression, prader willi syndrome, anxiety disorder, sclerosis, autism, fibromyalgia etc. Oxytocin is also responsible to elevate the levels of other hormones including growth hormone and testosterone while it declines the level of catabolic hormone such as cortisol due to which this hormone is also regarded as 'love hormone' and oxytocin is also responsible for exacerbate the orgasm and sexual desires. It is also accountable for uterine contraction during gestation. Elevated oxytocin level causes over sensitivity to emotions ${ }^{40}$.

Serotonin (5-HT) is a neurotransmitter which could also be referred as 'happiness hormone. Reduced serotonin level may cause mood chaos, anxiety and depression ${ }^{41}$. Its abnormal level may also cause poor sleep, elevated sensitivity to pain, migraine and headache. Imbalance of serotonin in the brain may cause obsessive thoughts and obsessive-compulsive disorder. If the level of serotonin gets elevated it may cause serotonin syndrome. Excessive serotonin in the body may cause nausea and vomiting, shivering and hyperthermia. Drugs that are accountable for the increased serotonin levels include Selective Serotonin Reuptake Inhibitor (SSRI), Serotonin Norepinephrine Reuptake Inhibitor (SNRI), MAO inhibitors, tricyclic antidepressants, cocaine etc.

Estrogens also play a vital role in women's mental health. Low levels of estrogen could cause menstrual exacerbation and mood lowering. General symptoms of low estrogen levels include vaginal dryness, irregularity in menstruation, hot flashes, and tenderness of breasts, fatigue, and osteoporosis due to decreased bone density and could also lead to infertility in women. Symptoms due to excessive estrogen are weight gain (especially in the waist, hips and thighs area), irregularity in menstrual cycles and mental illness which include panic attacks, anxiety, decreased sexual desires, agitation, depression, memory loss and irritability $\underline{\underline{42}}$.

Imbalance of testosterone in the body occurs due to PolyCystic Ovarian Syndrome (PCOS) in which the precipitation of excessive male hormone takes place, malfunctioning of adrenal glands, fluctuation of the other hormones, obesity, elevated blood sugar level, pregnancy, use of contraceptive pills and chronic illness. Increased testosterone level in women may exigence symptoms such as irregular menstruation, excessive growth of hair on the body and face, unusual growth of muscles etc., but if the 
level of this hormone declines it may be associated with painful intercourse, low libido, interrupted menstrual cycles, uneven sleeping patterns, weight gain and some mental illness such as mood changes, depression and anxiety $\stackrel{43}{ }$.

\section{Conclusion}

Behaviour of female depends on various factors, such as socio-environmental factors and physiological factors. Among the physiological factors, endogenous factors have wide role. Apart from sex hormones, there are many endogenous chemicals such as, serotonin, dopamine, oxytocin, endorphins, prolactin and cortisol that significantly affect women's mood and behaviour. Excessive low level of serotonin causes depression and aggression in female while its excessiveness can cause shivering, muscle's rigidity and diarrhoea. Dopamine is highly associated with pursuit of rewards and motivation. Feeling of pleasure, satisfaction, emotional responses and impulsive behaviour of women are inevitably associated with high level of dopamine. Oxytocin also influences women's behaviour. It is sometimes known as cuddle or love hormone, since its high level impel women to snuggle up or bond closely. Endorphins are endogenous opioid neuropeptides that act on opiate receptors and have tendency to reduce pain and boost pleasure. Endorphins are secreted in response to physical stress such as activities and exercise. Prolactin also influences women's behaviour. It stimulates breast development and milk production. Its excessiveness can cause disturbance in libido, irregular menstrual periods and excessive body and facial hair growth. The most influential hormone on stress behaviour is cortisol. Excess of cortisol in women can lead to aggressiveness in behaviour. It is also known as stress-hormone.

\section{References}

1. Steiner M, Dunn E, Born L. Hormones and mood: From menarche to menopause and beyond, Journal of Affective Disorders. 2003; 74(1):67-83. https://doi.org/10.1016/ S0165-0327(02)00432-9.

2. Anderson AK. Feeling emotional: the amygdala links emotional perception and experience, Social Cognitive and Affective Neuroscience. 2007; 2(2):71-72. https://doi. org/10.1093/scan/nsm022.
3. Stamova L, Anoshkina N, Nazirova A, Nikiforowa T. Dynamics of neuronal activity in the limbic system under emotional stress, Indian Journal of Science and Technology. 2016; 9(42):1-6. https://doi.org/10.17485/ijst/2016/v9i42/ 104303.

4. Van Dijk A, Klompmakers AA, Feenstra MGP, Denys D. Deep brain stimulation of the accumbens increases dopamine, serotonin, and noradrenaline in the prefrontal cortex, Journal of Neurochemistry. 2012; 123(6):897-903. https://doi.org/10.1111/jnc.12054.

5. Dolcos S, Katsumi Y, Dixon RA. The role of arousal in the spontaneous regulation of emotions in healthy aging: An FMRI investigation, Frontiers in Psychology. 2014; 5:681. https://doi.org/10.3389/fpsyg.2014.00681.

6. Kring AM, Gordon AH. Sex differences in emotion: Expression, experience, and physiology, Journal of Personality and Social Psychology. 1998; 74(3):686-703. https://doi.org/10.1037//0022-3514.74.3.686.

7. Halbreich U. Gonadal hormones and antihormones, serotonin and mood, Psychopharmacology Bulletin. 1990; 26(3):291-95.

8. Rajmohan V, Mohandas E. The limbic system, Indian Journal of Psychiatry. 2007; 49(2):132-39. https://doi. org/10.4103/0019-5545.33264.

9. Dfarhud D, Malmir M, Khanahmadi M. Happiness \&amp; Health: The Biological Factors- Systematic Review Article, Iranian Journal of Public Health. 2014; 43(11):1468-77.

10. Stiedl O, Pappa E, Konradsson-Geuken Å, Ögren SO. The role of the serotonin receptor subtypes 5-HT1A and 5-HT7 and its interaction in emotional learning and memory, Frontiers in Pharmacology. 2015; 6:162. https://doi.org/ 10.3389/fphar.2015.00162.

11. Patrick G, Combs G, Gavagan T. Initiating antidepressant therapy? Try these 2 drugs first, The Journal of Family Practice. 2009; 58(7):365-69.

12. Zhang S, Zeng X, Ren M, Mao X, Qiao S. Novel metabolic and physiological functions of branched chain amino acids: a review, Journal of Animal Science and Biotechnology. 2017; 8:10. https://doi.org/10.1186/s40104-016-0139-z.

13. Dearry A, Burnside B. Dopaminergic regulation of cone retinomotor movement in isolated teleost retinas: II. Modulation by gamma-aminobutyric acid and serotonin, Journal of Neurochemistry. 1986; 46(4):1022-31. https:// doi.org/10.1111/j.1471-4159.1986.tb00613.x.

14. Mishra A, Singh S, Shukla S. Physiological and functional basis of dopamine receptors and their role in neurogenesis: Possible implication for Parkinson's disease, Journal of Experimental Neuroscience. 2018. https:/doi.org/ 10.1177/1179069518779829.

15. Liu M, Luo J. Relationship between peripheral blood dopamine level and internet addiction disorder in 
adolescents: A pilot study, International Journal of Clinical and Experimental Medicine. 2015; 8(6):9943.

16. Schultz W. Dopamine reward prediction error coding, Dialogues in Clinical Neuroscience. 2016; 18(1):23-32.

17. Ježová D, Michajlovskij N, Kvetńanský R, Makara GB. Paraventricular and supraoptic nuclei of the hypothalamus are not equally important for oxytocin release during stress, Neuroendocrinology. 1993; 57(5):776-81. https://doi. org/10.1159/000126436.

18. Magon N, Kalra S. The orgasmic history of oxytocin: Love, lust, and labor. Indian journal of endocrinology and metabolism. 2011, 15, pp. 56-61. https://doi.org/ 10.4103/2230-8210.84851.

19. Varrassi G, Bazzano C, Edwards WT. Effects of physical activity on maternal plasma beta-endorphin levels and perception of labor pain, American Journal of Obstetrics and Gynecology. 1989; 160(3):707-12. https://doi.org/ 10.1016/S0002-9378(89)80065-1.

20. Hawkes CH. Endorphins: The basis of pleasure? Journal of Neurology, Neurosurgery, and Psychiatry. 1992; 55(4):247-50. https://doi.org/10.1136/jnnp.55.4.247.

21. Weizman R, Gil-Ad I, Hermesh H, Munitz H, Laron Z. Immunoreactive beta-endorphin, cortisol, and growth hormone plasma levels in obsessive-compulsive disorder, Clinical Neuropharmacology. 1990; 13(4):297-302. https:// doi.org/10.1097/00002826-199008000-00003.

22. Muccioli G, Ghè C, Di Carlo R. Distribution and characterization of prolactin binding sites in the male and female rat brain: Effects of hypophysectomy and ovariectomy, Neuroendocrinology. 1991; 53(1):47-53. https://doi.org/10.1159/000125696.

23. Ben-Jonathan N, Hnasko R. Dopamine as a prolactin (PRL) inhibitor, Endocrine Reviews. 2001; 22(6):724-63. https:// doi.org/10.1210/edrv.22.6.0451.

24. Majumdar A, Mangal NS. Hyperprolactinemia, Journal of Human Reproductive Sciences. 2013; 6(3):168-75. https:// doi.org/10.4103/0974-1208.121400.

25. Serri O, Chik CL, Ur E, Ezzat S. Diagnosis and management of hyperprolactinemia, CMAJ : Canadian Medical Association Journal (Journal de l'Association Medicale Canadienne). 2003; 169(6):575-81.

26. Gibney J, Smith TP, McKenna TJ. Clinical relevance of macroprolactin, Clinical Endocrinology. 2005; 62(6):633-43. https://doi.org/10.1111/j.1365-2265.2005. 02243.x.

27. Biller BM. Hyperprolactinemia, International Journal of Fertility and Women's Medicine. 2018; 44(2):74-77.

28. Laway B, Mir S. Pregnancy and pituitary disorders: Challenges in diagnosis and management, Indian Journal of Endocrinology and Metabolism. 2013; 17(6):996. https:// doi.org/10.4103/2230-8210.122608.
29. Lee DY, Kim E, Choi MH. Technical and clinical aspects of cortisol as a biochemical marker of chronic stress, BMB Reports. 2015; 48(4):209-16. https://doi.org/10.5483/ BMBRep.2015.48.4.275.

30. Roberts PJ, Coppola JC, Isaacs NW, Kennard O. Crystal and molecularstructureofcortisol(11 $\beta, 17 \alpha, 21$-trihydroxypregn4-ene-3,20-dione) methanol solvate, Journal of the Chemical Society. 1973; 6:774-81. https://doi.org/10.1039/ P29730000774.

31. Segerstrom SC. Stress, energy, and immunity: An ecological view, Current Directions in Psychological Science. 2007; 16(6):326-30. https://doi.org/10.1111/j.1467-8721.2007. 00522.x.

32. Hoyt LT, Zeiders KH, Ehrlich KB, Adam EK. Positive upshots of cortisol in everyday life, Emotion. 2016; 16(4):431-35. https://doi.org/10.1037/emo0000174.

33. Halbreich U, Endicott J. Possible involvement of endorphin withdrawal or imbalance in specific premenstrual syndromes and postpartum depression, Medical Hypotheses. 1981; 7(8):1045-58. https://doi.org/10.1016/ 0306-9877(81)90100-6.

34. Barry JA, Moran E, Parekh HS, Morewood T, Thomas M, Hardiman PJ. Prolactin and aggression in women with fertility problems, Journal of Obstetrics and Gynaecology : The Journal of the Institute of Obstetrics and Gynaecology. 2014; 34(7):605-10. https://doi.org/10.3109/01443615.201 4.901302 .

35. McEwen BS. Central effects of stress hormones in health and disease: Understanding the protective and damaging effects of stress and stress mediators, European Journal of Pharmacology. 2008; 583(2-3):174-85. https://doi. org/10.1016/j.ejphar.2007.11.071.

36. Michels A, Michels N. Addison disease: Early detection and treatment principles, American Family Physician. 2014; 89(7):563-68.

37. Seth S, Lewis AJ, Galbally M. Perinatal maternal depression and cortisol function in pregnancy and the postpartum period: A systematic literature review, BMC Pregnancy and Childbirth.2016;16(1):124.https://doi.org/10.1186/s12884016-0915-y.

38. Tost H, Alam T, Meyer-Lindenberg A. Dopamine and psychosis: Theory, pathomechanisms and intermediate phenotypes, Neuroscience and Biobehavioral Reviews. 2010; 34(5):689-700. https://doi.org/10.1016/j.neubiorev. 2009.06.005.

39. Sanna F, Bratzu J, Piludu MA, Corda MG, Melis MR, Giorgi O, Argiolas A. Dopamine, noradrenaline and differences in sexual behavior between roman high and low avoidance male rats: A microdialysis study in the medial prefrontal cortex, Frontiers in Behavioral Neuroscience. 2017; 11:108. https://doi.org/10.3389/fnbeh.2017.00108. 
40. Cyranowski JM, Hofkens TL, Frank E, Seltman H, Cai H-M, Amico JA. Evidence of dysregulated peripheral oxytocin release among depressed women, Psychosomatic Medicine. 2008; 70(9):967-75. https://doi.org/10.1097/ PSY.0b013e318188ade4.

41. Uphouse L. Pharmacology of serotonin and female sexual behavior, Pharmacology, Biochemistry, and Behavior. 2014; 121:31-42. https://doi.org/10.1016/j.pbb.2013.11.008.

42. Anastasiadis AG, Davis AR, Salomon L, Burchardt M, Shabsigh R. Hormonal factors in female sexual dysfunction,
Current Opinion in Urology. 2002; 12(6):503-07. https:// doi.org/10.1097/00042307-200211000-00011.

43. Barry JA, Hardiman PJ, Saxby BK, Kuczmierczyk A. Testosterone and mood dysfunction in women with polycystic ovarian syndrome compared to subfertile controls, Journal of Psychosomatic Obstetrics and Gynecology. 2011; 32(2):104-11 https://doi.org/10.3109/0 167482X.2011.568129. 